\title{
ROMANIAN
}

NEUROSURGERY

\author{
Vol. XXXV | No.1 March 2021
}

\section{Outcomes of surgical treatment for pituitary metastasis}

Dmytro S. Teslenko, Mykola O. Guk,

Olga Yu. Chuvashova 


\section{Outcomes of surgical treatment for pituitary metastasis}

\section{Dmytro S. Teslenko ${ }^{1}$, Mykola O. Guk ${ }^{2}$, Olga Yu. Chuvashova ${ }^{3}$}

\author{
1 Department of Neuroendoscopy and Craniofacial Surgery, SI \\ "Romodanov Neurosurgery Institute" NAMS of Ukraine, Kyiv, UKRAINE \\ 2 Department of Transsphenoidal Neurosurgery, SI "Romodanov \\ Neurosurgery Institute" NAMS of Ukraine, Kyiv, UKRAINE \\ 3 Department of Neuroradiology and Radioneurosurgery, SI \\ "Romodanov Neurosurgery Institute" NAMS of Ukraine, Kyiv, UKRAINE
}

\begin{abstract}
We analyzed the outcomes of surgical treatment for pituitary metastasis (PM) based on 35 observations. We registered the best estimates of resection radicalism in patients with minor PM, its inconsiderable spread ascending and descending from the diaphragm of the sella turcica, slightly bleeding metastasis, and no invading to the cavernous sinus. Positive changes in the PM patients' quality of life are chiefly associated with regressed visual impairment, local pain syndrome, and, to a lesser degree, oculomotor disorders. No post-operative regress or intensifying of hormonal impairments, such as diabetes insipidus and hypopituitarism, were seen. The age of patients, the time interval between the cancer diagnosis and the PM occurrence, control of the underlying disease, size of the tumour are the factors determining the life expectancy in PM patients.
\end{abstract}

\section{INTRODUCTION}

Metastatic pituitary lesion (hereinafter as PM - pituitary metastasis) is a very rare condition of the central nervous system (CNS), with its frequency reported by various authors ranging from 0.14 to $20 \%$ among all metastatic invasions to the brain. [1-3; 5;8-14]. No way all PM cases require surgical intervention, as the majority of PM diagnosed at autopsy $[2 ; 4 ; 6-8 ;]$ This evidence is associated with the commonly asymptomatic course: symptomatic PM incidence reaches only $7 \%$. The largest actual studies concerning PM demonstrate heterogeneous and ambiguous treatment outcomes for the patients with this disease. [1$3 ; 6 ; 8-14]$ Authors, though, state impossible to prolong the overall survival due to surgical intervention, concluding this based on the results of treatment of a significant number of patients having undergone surgery for PM. The number of observed patients operated on in these series ranges from 36 to 88 cases. $[1 ; 5 ; 8]$ In this study, we attempted to clarify what post-operative outcomes are expected for PM surgery.

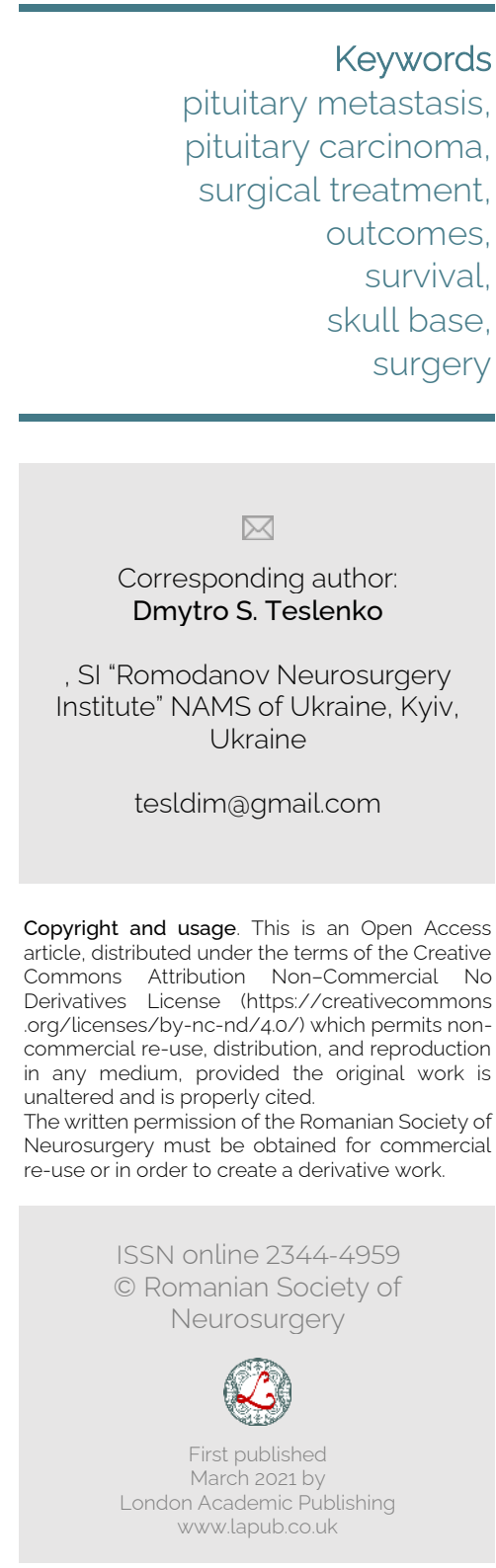


The purpose of the study is to improve the surgical tactics in PM via analyzing surgical treatment outcomes for the patients with this condition.

\section{MATERIAL AND METHOD}

The work is based on the analysis of surgical outcomes of 35 PM patients treated and monitored at the $\mathrm{SI}$ «Romodanov Neurosurgery Institute» NAMS of Ukraine, Kyiv, Ukraine from 2005 to 2015. The PM diagnosis was confirmed histologically in all 35 patients.

The study included 17 males (47.9\%) and 18 females $(52.1 \%)$. The mean age of the enrolled individuals was $56.4 \pm 10.4$ years: $60.8 \pm 2.4$ years for men, $52.2 \pm 2.1$ years for women. All 35 patients evidenced the symptomatic course of the disease. Among these 35 patients, the most common primary tumor was lung cancer (34.3\%), then in descending order, breast cancer $(22.9 \%)$, renal $(8.6 \%)$, rectal, prostate (all of 5.7\%). Diagnostic patterns for PM included clinical and laboratory, neuroimaging evaluations, and examinations of the related specialists (oncologist, ophthalmologist, endocrinologist, therapist).

27 of 35 patients (77.2\%) underwent microscopic PM removal. The observations were classified by type of approach, specifically, 7 (20.0\%) patients were performed PM transcranial removal, and 20 (57.2\%) transsphenoidal surgery. All cases of PM transcranial removal were performed from the subfrontal approach. In 8 patients (22.8\%) the removal was carried out by the endoscopic technique.

While the statistical analysis of the obtained material, the Mann-Whitney $U$ test was applied to independent samples, and the Wilcoxon signed-rank test to dependent samples to evaluate the probability of differences. Statistical correlation of parameters was estimated by the Spearman rank correlation test. The Kaplan-Meier method was applied to patient survival estimation. The Cox proportional hazard regression analysis was used to assess the prognosis.

\section{RESULTS AND DISCUSSION}

According to intraoperative findings and the results of post-operative tomographic control, total removal of PM took place in 7 patients (20.0\%), subtotal in 17 (48.6\%), partial in 9 (25.7\%), a biopsy was done in 2 patients $(5.7 \%)$. The most surgical radicalism was achieved in the patients who were operated on by transnasal endoscopic approach (4 cases). The lowest rates of total resection were registered in the group of patients who underwent microscopic transsphenoidal removal (2 observations). The distribution of PM patients by type and radicalism of the performed surgery is presented in Figure 1.

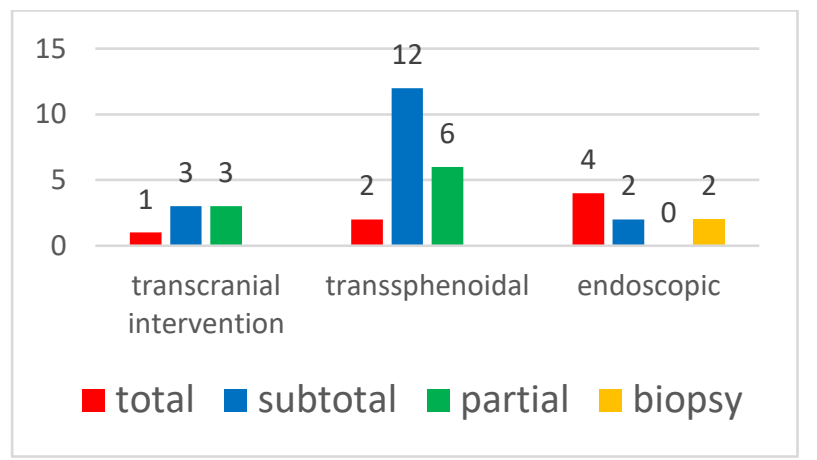

Figure 1. Observations distributed by the intervention radicalism and resection technique.

The Pairwise Correlation Method used for assessment of correlation between different factors and radicalism of operative intervention for PM showed an impact of the size of the metastasis, its spread, bleeding of tumor tissue, cavernous sinus lesions (all correlations significant at $p<0.05$ ). The best levels of radicalism were seen in smaller sizes, inconsiderable ascending and descending spread from the diaphragm of the sella turcica, slightly bleeding metastasis, and no invading to the cavernous sinus. The significance of these factors impact model in the ordinal regression analysis was $p=0.001 \quad(\chi 2=82.9)$. Visual disorders of varying severity were registered in 29 of 35 patients (82.9\%). In most patients, visual disorders were the main, and often the only, manifestation of the disease. The request to improve vision or at least stabilize current visual impairments was the main goal of surgical treatment for a considerable number of patients. Among 6 (17.1\%) of our patients with normal visual acuity and field of vision, no negative post-operative changes of visual functions were detected in any case. If the visual disorders were present primarily, the vision improvement was achieved in $79.3 \%$ cases (23 patients). Deterioration of vision was registered in 2 patients $(6.9 \%)$, no changes were observed in 33 patients (13.8\%). The Paired T-Test performed for comparison of two dependent samples (pre- and post-operative patients) showed the statistical significance of improvement of the visual disorders 
in our patients undergone operation $T=5.2$ at $p=$ 0.000 .

Among 18 patients with initial oculomotor disorders, post-operative improvement occurred in 9 cases (50.0\%); no dynamics was seen in 6 patients (33.3\%), deterioration occurred in 3 cases (16.7\%). In $4(23.5 \%)$ of 17 patients with no pre-operative oculomotor problems, post-operative oculomotor disorders appeared. Among 7 patients who developed or augmented oculomotor disorders after surgery, 2 cases of an isolated failure of sixth cranial nerves, 4 cases of third cranial nerves failure, and 1 case of combined lesions of third and sixth cranial nerves were observed.

Occurrence/deterioration of oculomotor functions in the study group after the endoscopic approach was equal to $14.7 \%$ of the total number of patients, and after microsurgical transsphenoidal and transcranial removal, these complications were registered more commonly, in $57.1 \%$ and $28.6 \%$ of cases, respectively. The obtained results are statistically significant $(p=0.041)$. Among those 4 patients who evidenced pre-operative dysfunction of trigeminal nerve branch, 2 individuals showed improvement $(50 \%)$ due to decreased numbness in the area of the nerve innervation, 2 patients (50\%) demonstrated no changes after surgery. In the postoperative period, trigeminal nerve failure appeared in 2 patients on the side of the tumor invasion into the cavernous sinus cavity.

Postoperatively, 14 patients with pre-operative hypopituitary disorders demonstrated no change in the severity of the pituitary failure. Deterioration of endocrine status, which was associated with the appeared hypothyroidism and hypocorticism, was observed in 3 patients. In the post-operative period, diabetes insipidus occurred in 4 patients who were not affected previously. In 20 patients with preoperative evidence of diabetes insipidus, no changes of this symptom were seen. The results which we obtained prove that endoscopic PM removal leads to a statistically significantly lower rate of endocrine disorders, including the development of diabetes insipidus, compared to microsurgical techniques ( $p$ $<0.05$ ). Onset or increase of hypopituitary disorders was also statistically significantly more common at microsurgical removal of PM ( $p<0.05)$. Among 6 patients operated on for PM, with the Karnofsky Performance Scale Index of the quality of life $>80$ in the early post-operative period, no worsening was registered in 5 patients, and in 1 individual the quality of life deteriorated. Among 19 patients with the Karnofsky Performance Scale Index of the quality of life equal to $70-80$ in the early post-operative period, the condition did not worsen in 12 patients, in 7 the quality of life improved. Among the 10 patients with the Karnofsky Performance Scale Index of the quality of life $<70$ in the early post-operative period, the condition did not change in 7 patients, in 3 the quality of life improved. The Paired T-Test comparison of two dependent samples (pre- and post-operative patients) showed the statistical significance of improving the quality of life of our operated patients $(T=-3.01$ at $p=0.005)$.

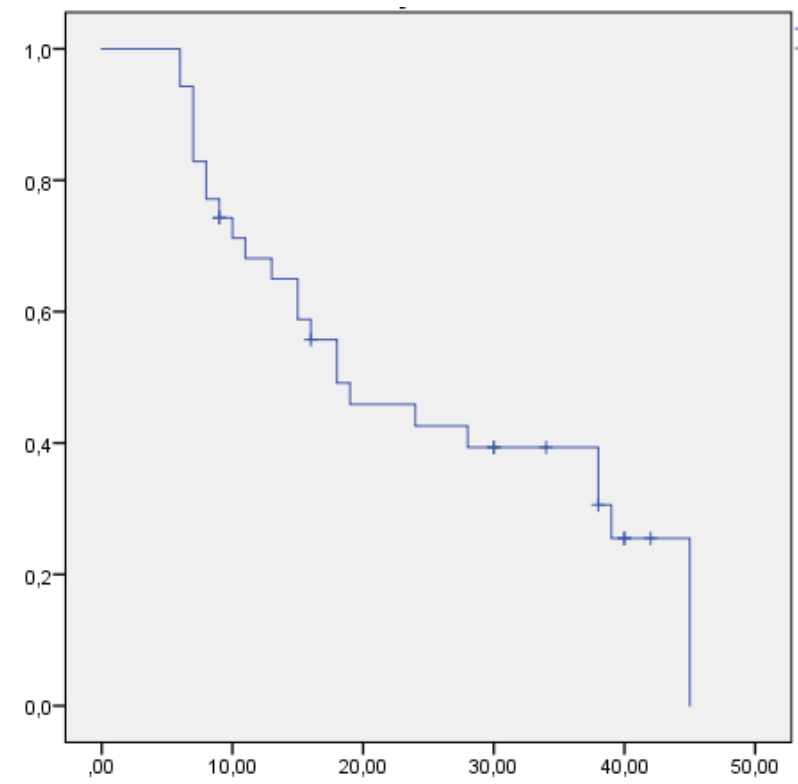

Figure 2. Overall survival of the patients operated on for PM (Kaplan-Meier).

All 35 observations were assessed with the Cox proportional hazards model regression analysis considering the available information on age, sex, the interval between diagnosis of the primary tumor and PM occurrence, the status of the primary lesion, PM maximum diameter, presence of other intracranial lesions, chemotherapy and/or radiation therapy. Our mathematical model was significant, $\mathrm{p}$ $=0.003(x 2=15.9)$. The following independent factors were associated with a better prognosis in PM patients (relative risk $<1, p<0.05$ ): younger age; female sex; late-onset pituitary metastasis; the smaller size of PM; radiation therapy underwent; control of the primary site condition. Sex of patients and the clinical group of medical examination 
considered as the factors with the most pronounced predictive properties in our patients. The average survival time in females was much longer than in males $(31.6 \pm 3.4$ months compared to $15.8 \pm 3.0$ months, respectively). The average survival time in the patients of the cancer clinical group IV was statistically significantly shorter than in patients of groups II and III (7.3 \pm 0.5 months compared to 27.3 \pm 3.8 and $32.3 \pm 4.3$ months, respectively) (Figure $3 \mathrm{~B}$ ).

A.

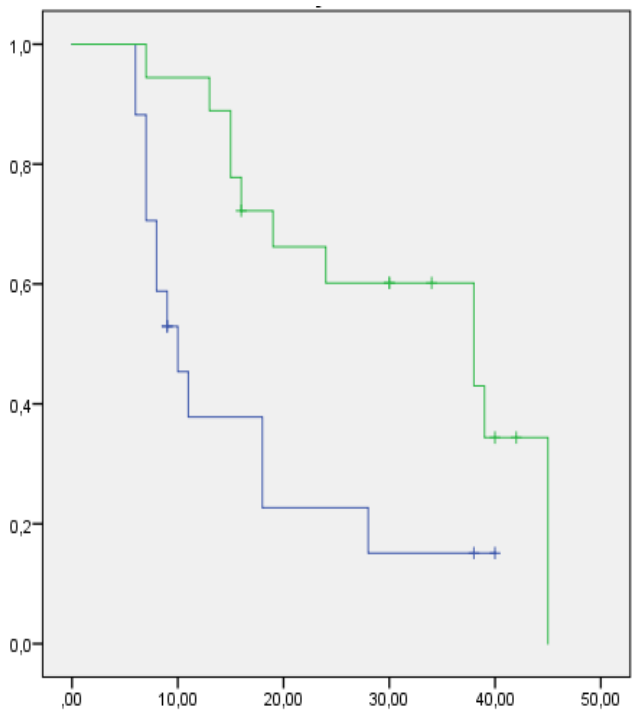

B.

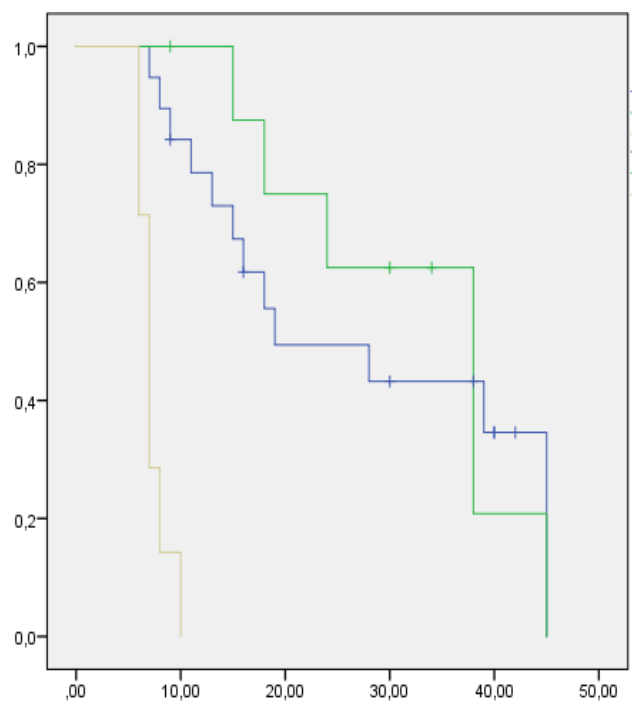

Figure 3. Survival curves of patients with PM (Kaplan-Meier): A. General survival curves obtained for different gender (male blue line/female - green line) groups of patients; B. General survival curves obtained for patients of different clinical groups (1st clinical group - blue line, $2 \mathrm{~d}$ clinical group - green line, 3rd clinical group - yellow line).
Thus, the analyzed treatment results of PM patients showed that the attempt to achieve an increased overall survival in PM patients compared to the available literature data failed. [ ] This result of the study indicates that the life expectancy in cancer patients with PM is primarily determined by the control of the underlying disease. In general, the life expectancy of PM patients was determined by the age of patients, the interval between the time of diagnosis of cancer and the occurrence of PM, control of the underlying disease, size of the tumor.

The individual tailoring principle should be followed when determining the indications to the surgical intervention for PM. If the tactics, technique of the surgical intervention, and the volume of removal are relevant, the quality of life in PM patients can be improved significantly. The dynamics of clinical manifestations in PM patients is more demonstrative in the long-term post-operative observations, that is associated either with a protracted recovery of neural structures of the sellar area after surgery, or the results of rehabilitation measures, as well.

Positive dynamics of the quality of life in PM patients is first and foremost associated with the regression of visual disturbances, local pain, and to a lesser extent oculomotor disorders. There is no postoperative regress or intensification of hormonal disorders, such as diabetes insipidus and hypopituitarism.

The prognosis for PM patients is poor, but it not attributable to the location, but due to the developing subsequent stages of primary cancer. We know that only 4 patients died of progressing pituitary lesions.

The short but definite survival time of PM cancer patients requires a change in the management strategy for these patients at all stages: from diagnosing to terminal care to ensure the long-term quality of life. In this context, we justify rationally in certain situations surgery and pituitary dysfunction treatment, including administration of gonadotropin, cortisol, thyroxine, which is rarely carried out in the complex management of patients with this condition.

\section{CONCLUSIONS}

1. The main indications for surgery for PM are present visual disorders, oculomotor impairments, pain syndrome caused by affected optic nerves and 
cavernous sinus, and no verified cancer diagnosis in the presence of clinical manifestations of the disease. These factors influence the choice of approach and methods of PM removal.

2. The radicalism of PM removal is determined by the size of the tumor, bleeding tumor tissue, metastatic invasion to the cavernous sinus, use of endoscopic technologies.

3. The best results of surgical treatment for PM can be achieved in younger patients, individuals with the controlled underlying oncological condition, with good pre-operative neurological status and quality of life, in those undergone radical removals of the tumor.

\section{REFERENCES}

1. Carey RM, Kuan EC, Workman AD, Patel NN, Kohanski MA, Tong CCL, Chen J, Palmer JN, Adappa ND, Brant JA. A Population-Level Analysis of Pituitary Carcinoma from the National Cancer Database. J Neurol Surg B Skull Base. 2020 Apr;81(2):180-186. doi: 10.1055/s-0039-1683435. Epub 2019 Mar 15. PMID: 32206537; PMCID: PMC7082172.

2. Kaltsas GA, Nomikos P, Kontogeorgos G, Buchfelder M, Grossman AB. Clinical review: Diagnosis and management of pituitary carcinomas. J Clin Endocrinol Metab. 2005 May;90(5):3089-99. doi: 10.1210/jc.20042231. Epub 2005 Mar 1. PMID: 15741248.

3. Raverot $G$, Burman $P$, McCormack $A$, Heaney $A$, Petersenn S, Popovic V, Trouillas J, Dekkers OM; European Society of Endocrinology. European Society of Endocrinology Clinical Practice Guidelines for the management of aggressive pituitary tumours and carcinomas. Eur J Endocrinol. 2018 Jan;178(1):G1-G24. doi: 10.1530/EJE-17-0796. Epub 2017 Oct 18. PMID: 29046323.

4. Ragel BT, Couldwell WT. Pituitary carcinoma: a review of the literature. Neurosurg Focus. 2004 Apr 15;16(4):E7. doi: 10.3171/foc.2004.16.4.8. PMID: 15191336.

5. Hansen TM, Batra S, Lim M, Gallia GL, Burger PC, Salvatori R, Wand G, Quinones-Hinojosa A, Kleinberg L, Redmond $\mathrm{KJ}$. Invasive adenoma and pituitary carcinoma: a SEER database analysis. Neurosurg Rev. 2014 Apr;37(2):27985; discussion 285-6. doi: 10.1007/s10143-014-0525-y. Epub 2014 Feb 14. PMID: 24526366; PMCID: PMC4322934.

6. Heaney AP. Clinical review: Pituitary carcinoma: difficult diagnosis and treatment [published correction appears in J Clin Endocrinol Metab. 2012 Mar;97(3):1064]. J Clin Endocrinol Metab. 2011;96(12):3649-3660. doi:10.1210/jc.2011-2031

7. Bengtsson D, Schrøder HD, Andersen $M$, Maiter $D$, Berinder $\mathrm{K}$, Feldt Rasmussen $\mathrm{U}$, Rasmussen ÅK, Johannsson G, Hoybye $C$, van der Lely AJ, Petersson M, Ragnarsson O, Burman P. Long-term outcome and MGMT as a predictive marker in 24 patients with atypical pituitary adenomas and pituitary carcinomas given treatment with temozolomide. J Clin Endocrinol Metab. 2015 Apr;100(4):1689-98. doi: 10.1210/jc.2014-4350. Epub 2015 Feb 3. PMID: 25646794.

8. In H, Bilimoria KY, Stewart AK, Wroblewski KE, Posner MC, Talamonti MS, Winchester DP. Cancer recurrence: an important but missing variable in national cancer registries. Ann Surg Oncol. 2014 May;21(5):1520-9. doi: 10.1245/s10434-014-3516-x. Epub 2014 Feb 7. PMID: 24504926.

9. Fassett DR, Couldwell WT. Metastases to the pituitary gland. Neurosurg Focus. 2004 Apr 15;16(4):E8. PMID: 15191337.

10. Goulart CR, Upadhyay S, Ditzel Filho LFS, Beer-Furlan A, Carrau RL, Prevedello LM, Prevedello DM. Newly Diagnosed Sellar Tumors in Patients with Cancer: A Diagnostic Challenge and Management Dilemma. World Neurosurg. 2017 Oct;106:254-265. doi: 10.1016/j.wneu.2017.06.139. Epub 2017 Jun 30. PMID: 28673886.

11. Zoli M, Mazzatenta D, Faustini-Fustini M, Pasquini E, Frank G. Pituitary metastases: role of surgery. World Neurosurg. $2013 \quad$ Feb;79(2):327-30. doi: 10.1016/j.wneu.2012.03.018. Epub 2012 Apr 2. PMID: 22480977.

12. Post KD. Pituitary metastases: what is the role of surgery? World Neurosurg. 2013 Feb;79(2):251-2. doi: 10.1016/j.wneu.2012.05.006. Epub 2012 May 24. PMID: 22634465.

13. Ariel D, Sung H, Coghlan N, Dodd R, Gibbs IC, Katznelson L. Clinical characteristics and pituitary dysfunction in patients with metastatic cancer to the sella. Endocr Pract. 2013 Nov-Dec;19(6):914-9. doi: 10.4158/EP12407.OR. PMID: 23757610.

14. Popławska-Kita A, Wielogórska M, Poplawski Ł, Siewko K, Adamska A, Szumowski P, Myśliwiec P, Myśliwiec J, Reszeć J, Kamiński G, Dzięcioł J, Tobiaszewska D, Szelachowska M, Krętowski AJ. Thyroid carcinoma with atypical metastasis to the pituitary gland and unexpected postmortal diagnosis. Endocrinol Diabetes Metab Case Rep. 2020 Mar 13;2020:19-0148. doi: 10.1530/EDM-190148. Epub ahead of print. PMID: 32168468; PMCID: PMC7077587. 